adverse events of interest requires consideration of how these may present in neonatal/paediatric patients.

Conclusions In order to support the protocol development with regards to neonatal/paediatric drug safety a dual competence in both paediatrics and drug safety is required. This review provides an overview of the practical aspects related to neonatal/paediatric drug safety during protocol development.

Disclosure(s) Nothing to disclose

\section{P12 PUBLIC ANTIBIOTIC AWARENESS CAMPAIGN ORGANISED BY GOVERNMENT SIGNIFICANTLY REDUCED INAPPROPRIATE ANTIBIOTIC USE IN PAEDIATRIC PRIMARY CARE SETTINGS}

\author{
1,2M Bajcetic*, 'M Lazic, ${ }^{2}$ Lukic, ${ }^{2} \mathrm{D}$ Rajkovic. 'Department of Clinical Pharmacology, \\ School of Medicine, Univeristy of Belgrade; ${ }^{2}$ Second Serbia Health Project, Ministry of Health \\ Republic of Serbia, Belgrade, Serbia
}

10.1136/archdischild-2019-esdppp.51

Serbia, like most other countries in southern Europe, has struggled with high rate of antibiotic consumption. Previous results showed that most of antibiotics were prescribed inappropriately, mainly for influence - like illness ${ }^{1}$. The first short term media antibiotic awareness campaign (AAC) was held in 2011. and 2014. respectively. Shortly after, Ministry of Health, Republic of Serbia in November 2015 has started with public AAC simultaneously across all regions of Republic of Serbia.

Apart from media, the campaign included education, producing national guidelines, as well as regulations. The education goals for public (preschool and school children, parents, pregnant women, students) and healthcare professionals (paediatricians, nurses, pharmacists, etc.) was based on results of analysis of antibiotic consumption in children from 2007.to 2014. which methodology was published previously. ${ }^{1}$ Media campaign included public relations activities, press conferences, billboards, printed materials, etc.

In 2017. prescribing rates of antibiotics per 1000 children in primary care settings were decreased by $18 \%$ comparing to 2011., after first and by $12 \%$ comparing to 2014 . when second short term media campaign was performed. After the third, government organised public AAC, prescribing rates of antibiotics per 1000 children in primary care settings were decreased by $6 \%$ for only one year (2017 vs. 2016) in all age groups from 2 months up to 17 years. Significant decrease of prescribed rate of antibiotics per 1000 children during 2017. was recorded for indication with policy of delayed or no antibiotic prescription recommended by guidelines. Seasonal oscillations showed that highest prescribing rate during the winter months (I and the IV quarter) of 2017. is in line with the lowest prescribing rate during the summer months (II and the III quarter) from 2007. up to 2013.

We can conclude that continuous antibiotic awareness campaign supported by state government is the best way to achieve successful results.

\section{REFERENCES}

1. Bozic B, Bajcetic M. Use of antibiotics in paediatric primary care settings in Serbia. Arch Dis Child 2015 Oct;100(10):966-9.

Disclosure(s) Nothing to disclose

\section{P13 \\ SERIOUS ADVERSE REACTIONS AND OFF LABEL AND UNLICENSED DRUG USE IN CHILDREN - DECADE OF PHARMACOVIGILANCE STUDY IN SERBIA}

1,2M Bajcetic*, 2,3J Joksimovic. 'University Children's Hospital; ${ }^{2}$ Department of Clinical Pharmacology, School of Medicine, Univeristy of Belgrade; ${ }^{3}$ Medicines and Medical Devices Agency of Serbia, Belgrade, Serbia

\subsection{6/archdischild-2019-esdppp.52}

Background Off label (OL) and unlicensed (UL) drug use in children is a widespread global problem. Previous study showed that only $66 \%$ of all available drugs for children is with licence in Serbia. ${ }^{1}$ Data on safety of medicines in children remain lacking, so the key intervention for the effective use of medicine is safety monitoring. Therefore, the aim of this study is to evaluate safety implication of OL and UL drug use in children up to 12 years old.

Method We conducted a retrospective study based on reports of suspected adverse reactions (ADRs) collected from 2008. to 2018. by Medicines and Medical Devices Agency of Serbia, using the Medical Dictionary for Regulatory Activities and organized by System Organ Class. Sources of information about medicines including vaccines (license, drug formulation, etc.) are the Summary of Product Characteristics and Serbia's official drug registry.

Results Within 10 years, we observed 1595 ADRs. Vaccines, antineoplastic and antimicrobial drugs were the most frequently pharmacotherapeutic subgroups involved. Out of total number of observed ADRs, 433 (28\%) were serious; 189 of them led to hospitalization, 31 to life threatening conditions and 7 were fatal. More than a half $(63 \%)$ of serious ADRs were detected in children for the age group of 28 days - 23 months, followed by the age group of 2 to 11 years (34\%) and finally by age group of 0 to 27 days (3\%). Serious ADRs were detected in boys (55\%) as well as in girls (45\%). Out of total number of registered only 3\% (46) of ADRs were associated with off-label use; 18 of them were serious, 7 led to life threatening conditions and 3 were fatal.

Conclusion This research provided new insight on the factors such as OL and UL use, that might increase the risk of serious ADRs in children.

\section{REFERENCES}

1. Božić B, Stupar S, Stupar D, Babić U, Bajčetić M. Availability of pediatric-evaluated formulations in Serbia. Indian J Pharmacol 2017 Mar-Apr;49(2):189-193

Disclosure(s) Nothing to disclose

\section{P14 PHARMACOVIGILANCE IN PEDIATRIC PATIENTS: THE CHALLENGE OF IDENTIFYING NEW SIGNAL}

${ }^{1} \mathrm{~N}$ Kronenfeld, ${ }^{2} \mathrm{~S}$ Gamsu, ${ }^{2} \mathrm{H}$ Flor-Hirsch, ${ }^{2} \mathrm{~N}$ Agajani, ${ }^{2} \mathrm{R}$ Sheinberg, ${ }^{2} \mathrm{~L}$ Ben-Nun, ${ }^{2,3} \mathrm{M}$ Goldman, ${ }^{1,3} \mathrm{M}$ Berkovitch*. ${ }^{1}$ Clinical Pharmacology and Toxicology; ${ }^{2}$ Division of Pediatrics, Assaf Harofeh Medical Centre, Zerifin; ${ }^{3}$ Sackler Faculty of Medicine, Tel-Aviv University, Tel Aviv, Israel

\subsection{6/archdischild-2019-esdppp.53}

Introduction The importance of reporting adverse drug reactions (ADRs) is well known. However, the reporting rate is very low, and therefore, identifying new signal is challenging. Objective To create an interventional program in order to improve reporting rate and trying to identify new signal. 